\title{
Evaluation of the Single-Population Lattice Boltzmann Method for One-Dimensional Compressible Flows
}

\author{
Samuel J. Mitchell* \\ Imperial College London, United Kingdom \\ Sina Stapelfeldt ${ }^{\dagger}$ \\ Imperial College London, United Kingdom \\ Ricardo Puente \\ Imperial College London, United Kingdom
}

\begin{abstract}
In this work the stability and accuracy of the one-dimensional single-population Lattice Boltzmann Method (LBM) is studied in its application to compressible flow regimes. A linear stability analysis framework using the von Neumann method is developed, framed in terms of flow regime parameters. In order to validate this analysis and study the behavior of errors with respect to different simulation parameters, two academic test cases are presented. A shock tube configuration is used to assess the shock capturing capabilities. A high subsonic convergentdivergent nozzle is used to evaluate the error dependence on grid size. This test case requires the inclusion of an additional source term to the Boltzmann equation, and a methodology is proposed for the implementation of physically motivated source terms. The proposed numerical method is shown to be accurate, matching theoretical error trends. Through stability analysis, bounds for the simulation parameters resulting in a stable simulation are given, which can inform best practices in the application of $\mathrm{LBM}$ to these regimes.
\end{abstract}

\section{Nomenclature}

$f=$ Particle distribution function

$f^{e q}=$ Equilibrium distribution function

$i=$ Space discretization index

$M=$ Fluid Mach number

$n=$ Time discretization index

$p \quad=\quad$ Fluid pressure

$R e_{g}=$ Grid Reynolds number

$T=$ Fluid temperature

$u=$ Fluid velocity

$\Delta x=$ Grid size

$\Delta t \quad=$ Time step

$\kappa=$ Advective Courant number

$\xi \quad=$ Particle velocity

$\theta=$ Product of fluid temperature and gas constant

$\rho \quad=\quad$ Fluid density

\section{Introduction}

The Lattice Boltzmann Method (LBM) is a computational fluid dynamics method which provides an efficient means to model a wide range of low-speed fluid dynamics problems, ranging from flows around insect wings [1] to urban

\footnotetext{
*PhD Candidate, Department of Mechanical Engineering, Imperial College London, South Kensington, London, SW7 $2 \mathrm{AZ}$.

${ }^{\dagger}$ Research Fellow, Department of Mechanical Engineering, Imperial College London, South Kensington, London, SW7 2AZ

${ }^{\ddagger}$ Research Fellow, Department of Mechanical Engineering, Imperial College London, South Kensington, London, SW7 2AZ
} 
city environments [2]. It however contains limitations that until recently have left the high-speed compressible flows found in aerospace and turbomachinery out of its reach. One of the main limitations is commonly referred to as the compressibility error, which limits the validity of standard LBM to incompressible and isothermal flows. This stems from the order of discretization of the equilibrium distribution function in the particle velocity space. The discretization route for standard LBM velocity sets, such as D1Q3 and D2Q9 [3], introduces an error term to the viscous momentum flux tensor which scales proportionally to the cube of the mean fluid velocity [4]. A method with a higher order of discretization can however recover the Navier-Stokes-Fourier (NSF) equations without this error, thus enabling accurate simulation of compressible flows with energy conservation.

There are two general approaches to deriving higher-order LB methods. The first is the single-population approach, where one population of particles with distribution function $f$ recovers all three macroscopic conservation equations. This was first presented by Alexander and Chen [5], and later extended by Kataoka and Tsutahara [6], who introduced an arbitrary specific heat ratio. The single population methods are advantageous in terms of implementation due to the intrinsic coupling of viscous heating and compression work between the momentum and energy equations [3]. However, concerns have been raised in existing research about their relative instability, especially in three dimensions [3] [7].

The second approach is the two-population, for which one population $f$ recovers the continuity and momentum equations, and another population $g$ recovers the energy equation. The first two-population method [8] provided improvements in stability over the early single-population methods, although was limited to thermal flows of low Mach number [9]. In addition, the need for non-local source terms to couple the viscous heating and compression work of the momentum and energy equations increases difficulty of implementation [3]. Guo et al. [10] and Karlin et al. [11] provided methods with improved implementation by local coupling of the energy equation. Methods valid for compressible flows have since been developed [7, 12], with the introduction of higher order velocity sets. Two-population methods may posses improved stability, and allow an arbitrary Prandtl number [3]. They are however more complicated to implement and require a greater amount of discrete velocities in comparison to the single-population method.

Another limitation to high-speed flows true to both of the above approaches is that instability is often encountered when modelling shocks. Although the standard LBM space-time scheme; collision-streaming, can be implemented with high efficiency [3], it contains insufficient dissipation to model discontinuities. This has led some existing researchers to replace it with finite difference schemes with increased dissipation, such as in the single-population methods of Dellar [9], and Kataoka and Tsutahara [6]. Furthermore, Gan et al. [13] and Chen et al. [14] introduce an additional source term to increase numerical dissipation around discontinuities. Frapolli et al. [15] have successfully retained the use of the collision-streaming scheme, along with its efficiency advantages, with use of the entropic collision operator.

The single-population method is attractive due to its relative ease of implementation and lower amount of discrete velocities, which may offer benefits in computational efficiency. The aim of this study is to evaluate both stability and accuracy of this method, to determine its potential for future use of modelling scale-resolved compressible flows. The existing single-population method with arbitrary specific heat ratio presented by Dellar [9] is considered, which contains a higher order particle velocity discretization and a finite difference space-time scheme. Firstly, a theoretical analysis of linear stability for this LB method using the von Neumann technique is presented. Existing stability analyses of the LBM have been presented by Sterling and Chen [16], and Lallemand and Luo [17], who focussed on LB methods for incompressible flow. Siebert et al. [18] presented a stability analysis study for a range of single-population methods for compressible flow. To the authors' knowledge, analysis of the single-population method with arbitrary specific heat ratio described above has however not yet been reported in existing research. The analysis proposed in this study shall present the stability of the method in terms of the Mach and Reynolds number of the considered flow, which shall prove beneficial for application to engineering test cases that are characterized by these quantities. The analysis shall then be applied to the conditions of the nozzle test case to be studied in the proceeding sections, and the resulting stability maps shall highlight the limitations on method parameters.

Next, two Euler test cases are evaluated experimentally, in order to test the capabilities of the method to model high speed flows, and the effect of the implicitly added viscosity is quantified. The first is Sod's shock tube [19], which tests the ability of the space-time scheme to capture discontinuities. The second is the quasi one-dimensional convergent-divergent nozzle. For this case, the additional required source term is included in the discrete Boltzmann equation. The nozzle case enables testing over a range of Mach numbers within the compressible regime. Assuming the nozzle flow remains subsonic, the solution for this test case is smooth, which allows evaluation of the spatial order of the error.

Accepted manuscript: AIAA SciTech 2021 Forum, https://doi.org/10.2514/6.2021-1663

Copyright (c) 2021 by the American Institute of Aeronautics and Astronautics, Inc. All rights reserved. 


\section{Lattice Boltzmann Method for Compressible Flows}

Equation (11) shows the continuous Boltzmann equation, which governs the evolution of the particle distribution function $f(\boldsymbol{x}, \boldsymbol{\xi}, t)$. Its value represents the density of particles with velocity $\boldsymbol{\xi}$, at position $\mathbf{x}$ and time $t$. The collision operator on the RHS drives the relaxation of $f$ towards an equilibrium state with distribution $f^{e q}$ [3]. The single relaxation time Bhatnagar-Gross-Krook (SRT-BGK) collision operator has been adopted here, which describes linear relaxation at a rate determined by the relaxation time $(\tau)$. All quantities used in sections [II [V] are presented in non-dimensional form.

$$
\begin{gathered}
\frac{\partial f}{\partial t}+\boldsymbol{\xi} \cdot \boldsymbol{\nabla} f=-\frac{1}{\tau}\left(f-f^{e q}\right) \\
\int f d \boldsymbol{\xi}=\rho, \quad \int f \boldsymbol{\xi} d \boldsymbol{\xi}=\rho \boldsymbol{u}, \quad \frac{1}{2} \int f|\boldsymbol{\xi}|^{2} d \boldsymbol{\xi}=\rho E
\end{gathered}
$$

Macroscopic fluid properties such as mass density $(\rho)$, mean velocity $(\mathbf{u})$ and total energy $(E)$ can be obtained from integrals of $f$ over the particle velocity $(\xi)$ space. These integrals are often referred to as moments of an order in $\boldsymbol{\xi}$, for example the zeroth to second order moments shown in Eq. (2). Integration of the Boltzmann equation over $\boldsymbol{\xi}$, from the zeroth to second order in $\xi$, will recover the Navier-Stokes-Fourier equations $(3)-(5)$.

$$
\begin{gathered}
\frac{\partial \rho}{\partial t}+\boldsymbol{\nabla} \cdot(\rho \boldsymbol{u})=0 \\
\frac{\partial \rho \boldsymbol{u}}{\partial t}+\boldsymbol{\nabla} \cdot(\rho \boldsymbol{u} \boldsymbol{u})=-\boldsymbol{\nabla} p+\boldsymbol{\nabla} \cdot \boldsymbol{\sigma} \\
\frac{\partial \rho E}{\partial t}+\boldsymbol{\nabla} \cdot(\rho \boldsymbol{u} E)=\boldsymbol{\nabla} \cdot(-p \boldsymbol{u}+\boldsymbol{u} \cdot \boldsymbol{\sigma})+\boldsymbol{\nabla} \cdot(\lambda \boldsymbol{\nabla} T)
\end{gathered}
$$

Pressure ( $p$ ) may be linked to density and temperature $(T)$ by assuming the Ideal Gas Law. The viscous component of the momentum flux tensor $(\sigma)$ can be determined using the Chapman-Enskog expansion [20]. During this, $f$ is approximated as the sum of the equilibrium $\left(f^{e q}\right)$ and a small first-order non-equilibrium $\left(\epsilon f^{(1)}\right)$ perturbation, and Eq. (6) can be obtained, with fluid shear viscosity $(\mu)$, bulk viscosity $\left(\mu_{B}\right)$ and thermal conductivity $(\lambda)$ as functions of the relaxation time.

$$
\boldsymbol{\sigma}=\mu\left[(\boldsymbol{\nabla} \boldsymbol{u})+(\boldsymbol{\nabla} \boldsymbol{u})^{T}-\frac{2}{3}(\boldsymbol{\nabla} \cdot \boldsymbol{u}) \boldsymbol{I}\right]+\mu_{B}(\boldsymbol{\nabla} \cdot \boldsymbol{u}) \boldsymbol{I}
$$

\section{A. Particle Velocity Discretization}

The first stage of preparing Eq. (1) for numerical solution is discretization of $f$ and $f^{e q}$ in the $\xi$ space. The continuous velocity integral moments such as those in Eq. (2) are replaced by sums of a set of $\alpha=0,1, \ldots N$ discrete populations $f_{\alpha}(x, t)$, each with a corresponding particle velocity $\xi_{\alpha}$ and equilibrium $f_{\alpha}^{e q}$. The evolution of each discrete population is governed by the discrete velocity Boltzmann equation (DVBE) [3]. Equation (7) shows the DVBE in one-dimensional space $x$ and particle velocity $\xi_{\alpha}$.

The DVBE will correctly recover the macroscopic equations (3)-(5) given that $f^{e q}$ is discretized in $\xi$ such that the discrete moments of $f_{\alpha}^{e q}$ equate to the corresponding continuous moments of $f^{e q}$ to a high enough order in $\xi$. There are several established discretization approaches, such as the Hermite series expansion [21], and direct moment-matching [9. 13]. For this study, the moment-matching discretization presented by Dellar for the single-population method [9] is chosen, which also contains Kataoka and Tsutahara's method [6] of achieving an arbitrary specific heat ratio $(\gamma)$ by including fixed internal energy coefficients to the particle distributions.

Central to the moment-matching discretization is the fact that in order for the NSF equations to be completely recovered by the DVBE, $f_{\alpha}^{e q}$ moment constraints up to the fourth order in $\xi$ must be satisfied. In one-dimensional space, considering also the adaption for arbitrary specific heat ratio, this results in a set of seven moment constraint equations [9], meaning at least $N=7$ discrete populations are required. Dimensionless discrete velocities are chosen as $\xi_{i}=[-3,-2,-1,0,1,2,3]$, and following common LBM notation, this discretization shall be referred to as D1Q7.

$$
\frac{\partial f_{\alpha}}{\partial t}+\xi_{\alpha} \frac{\partial f_{\alpha}}{\partial x}=-\frac{1}{\tau}\left(f_{\alpha}-f_{\alpha}^{e q}\right)
$$

Accepted manuscript: AIAA SciTech 2021 Forum, https://doi.org/10.2514/6.2021-1663

Copyright $\odot 2021$ by the American Institute of Aeronautics and Astronautics, Inc. All rights reserved. 
The seven moment constraint equations may be organized into the matrix system shown in Eq. (8). As is common with LBM, temperature $(T)$ and ideal gas constant $(R)$ are expressed as one quantity $\theta=R T$. The derivation of each moment can be found in [9]. The zeroth, first and second moments ensure correct recovery of mass density, momentum and the equilibrium component of the momentum flux tensor respectively. The third has been derived by Dellar through Chapman-Enskog analysis to ensure that the correct form of the viscous momentum flux tensor shown in Eq. (6) is recovered. The fourth and fifth recover total energy density and the equilibrium energy flux vector respectively. The sixth was again derived through Chapman-Enskog analysis and ensures the correct viscous contribution to the energy flux tensor. This matrix system may now be solved to obtain $f_{\alpha}^{e q}$. This discretization ensures complete recovery of Eqs. [3]-[5, with fluid properties of $\mu=\tau \rho \theta, \mu_{b}=\tau \rho \theta\left(\frac{5}{3}-\gamma\right)$ and $\lambda=\tau \rho \theta \frac{\gamma}{(\gamma-1)}$ [9].

$$
\underbrace{\left[\begin{array}{ccccccc}
1 & 1 & 1 & 1 & 1 & 1 & 1 \\
-3 & -2 & -1 & 0 & 1 & 2 & 3 \\
9 & 4 & 1 & 0 & 1 & 4 & 9 \\
-27 & -8 & -1 & 0 & 1 & 8 & 27 \\
9 & 4 & 2 & 4 & 2 & 4 & 9 \\
-27 & -8 & -2 & 0 & 2 & 8 & 27 \\
81 & 16 & 2 & 0 & 2 & 16 & 81
\end{array}\right]}_{\mathbf{C}} \underbrace{\left[\begin{array}{l}
f_{0}^{e q} \\
f_{1}^{e q} \\
f_{2}^{e q} \\
f_{3}^{e q} \\
f_{4}^{e q} \\
f_{5}^{e q} \\
f_{6}^{e q}
\end{array}\right]}_{\mathbf{f}}=\rho \underbrace{\left[\begin{array}{c}
1 \\
u \\
u^{2}+\theta \\
u^{3}+3 \theta u \\
u^{2}+\frac{2 \theta}{\gamma-1} \\
u^{3}+\frac{2 \theta u \gamma}{\gamma-1} \\
u^{4}+\theta u^{2} \frac{(5 \gamma-3)}{(\gamma-1)}+\frac{2 \theta^{2} \gamma}{(\gamma-1)}
\end{array}\right]}_{\mathbf{m}}
$$

\section{B. Space-Time Discretization}

The standard space-time discretization approach for LBM employs the method of characteristics and forward Euler temporal integration to the DVBE, resulting in the efficient, second-order collision-streaming scheme [3]. However for LB methods using the BGK collision operator, the low dissipation of this scheme leads to instability when modelling discontinuous flows. In existing research this problem was alleviated by replacing collision-streaming with alternative finite difference schemes [6, 9, 22]. Similarly to Dellar, the Beam-Warming scheme is used for this study [23] due to its greater dissipation compared with collision-streaming. Additionally, the time step for this scheme may be adjusted if required to achieve stability.

Equations (9)-(10) show the Beam-Warming discretization of the DVBE for an equispaced grid of $\Delta x$ and time step $\Delta t$, where integer indices $i$ and $n$ refer to the discrete space-time position $(i \Delta x, n \Delta t)$. The advective Courant number $\kappa=\frac{|\xi|_{\max } \Delta t}{\Delta x}$ is introduced, for which the critical wave speed is the largest discrete velocity $|\xi|_{\max }=3$. Equation $[9]$ is written in the upwind direction for negative wave speeds $(\xi<0)$, and Eq. [10) for $\xi>0$. The collision operator is here included directly as a forward Euler integration, as opposed to Dellar's method based on the Strang-splitting procedure [9]. By doing this, the amount of numerical operations per time step is reduced by removing the need for intermediate solution stages. It should however now be expected to lose the stability benefits the Strang-splitting scheme may offer.

$$
\begin{array}{r}
f_{\alpha, i}^{n+1}=f_{\alpha, i}^{n}+\frac{1}{2} \xi_{\alpha} \frac{\kappa}{|\xi|_{\max }}\left(3 f_{\alpha, i}^{n}-4 f_{\alpha, i+1}^{n}+f_{\alpha, i+2}^{n}\right) \\
+\frac{1}{2}\left(\xi_{i} \frac{\kappa}{|\xi|_{\max }}\right)^{2}\left(f_{\alpha, i}^{n}-2 f_{\alpha, i+1}+f_{\alpha, i+2}^{n}\right)-\frac{\Delta t}{\tau}\left(f_{\alpha, i}^{n}-f_{\alpha, i}^{e q}\right) \\
f_{\alpha, i}^{n+1}=f_{\alpha, i}^{n}-\frac{1}{2} \xi_{\alpha} \frac{\kappa}{|\xi|_{\max }}\left(3 f_{\alpha, i}^{n}-4 f_{\alpha, i-1}^{n}+f_{\alpha, i-2}^{n}\right) \\
\left.+\frac{1}{2}\left(\xi_{\alpha}^{n} \frac{\kappa}{|\xi|_{\max }}\right)^{2}\left(f_{\alpha, i}^{n}-2 f_{\alpha, i-1}^{n}+f_{\alpha, i-2}^{n}\right)-\frac{\Delta t}{\tau}\left(f_{\alpha, i}^{n}-f_{\alpha, i}^{e q}\right)\right)
\end{array}
$$

\section{Formulation for Quasi One-Dimensional Flow}

The methodology covered in this section is required for modelling of the quasi one-dimensional convergent-divergent nozzle test case. Assuming idealized conditions of cross-sectionally uniform, inviscid flow, it is possible to represent a three-dimensional convergent-divergent nozzle flow in one-dimensional space [24]. A one-dimensional solver with

Accepted manuscript: AIAA SciTech 2021 Forum, https://doi.org/10.2514/6.2021-1663

Copyright (C 2021 by the American Institute of Aeronautics and Astronautics, Inc. All rights reserved. 
two modifications may be used to model the quasi one-dimensional conservation equations (11)- (13). The first is the inclusion of area extensive conservative variables. The second is the addition of a source term $S=p \frac{d A}{d x}$ to the momentum equation, which is necessary to represent the effect of area variation.

$$
\begin{gathered}
\frac{\partial \rho A}{\partial t}+\frac{\partial \rho u A}{\partial x}=0 \\
\frac{\partial \rho u A}{\partial t}+\frac{\partial \rho u^{2} A}{\partial x}=-\frac{\partial A p}{\partial x}+p \frac{d A}{\partial x} \\
\frac{\partial \rho E A}{\partial t}+\frac{\partial \rho E u A}{\partial x}=-\frac{\partial p u A}{\partial x}
\end{gathered}
$$

The DVBE may be modified to approximate Eqs. (11)- 13 by using area extensive populations, and adding a discrete source term $S_{i}$, as in Eq. (14). Note that since Eqs. (11)-(13) describe an inviscid fluid, Eq. (14) does not provide exact recovery of this system because of the viscous contribution from the collision operator. Since $\mu=\tau \rho \theta$, maintaining a small relaxation time will minimise this error.

$$
\frac{\partial A f_{\alpha}}{\partial t}+\xi_{\alpha} \frac{\partial A f_{\alpha}}{\partial x}=-\frac{1}{\tau}\left(A f_{\alpha}-A f_{\alpha}^{e q}\right)+S_{\alpha}
$$

The source term $S$ imposes an additional momentum on the fluid, but has no influence on the mass and energy conservation equations. It is therefore the second order particle velocity moment of $S_{\alpha}$ which must recover this momentum source, as shown in Eq. (16). All other moments up to the fourth order must equal zero to ensure there is no undesired influence on the macroscopic equations. This allows the formation of the matrix system of moment equations (15), which may then be solved to obtain $S_{\alpha}$.

$$
\underbrace{\left[\begin{array}{ccccccc}
1 & 1 & 1 & 1 & 1 & 1 & 1 \\
-3 & -2 & -1 & 0 & 1 & 2 & 3 \\
9 & 4 & 1 & 0 & 1 & 4 & 9 \\
-27 & -8 & -1 & 0 & 1 & 8 & 27 \\
9 & 4 & 2 & 4 & 2 & 4 & 9 \\
-27 & -8 & -2 & 0 & 2 & 8 & 27 \\
81 & 16 & 2 & 0 & 2 & 16 & 81
\end{array}\right]}_{\mathbf{C}} \underbrace{\left[\begin{array}{c}
S_{0} \\
S_{1} \\
S_{2} \\
S_{3} \\
S_{4} \\
S_{5} \\
S_{6}
\end{array}\right]}_{\mathbf{s}_{\mathbf{i}}}=\underbrace{\left[\begin{array}{c}
0 \\
-p \frac{d A}{d x} \\
0 \\
0 \\
0 \\
0 \\
0
\end{array}\right]}_{\mathbf{m}}
$$

\section{Linear Stability}

Stability analysis is a useful tool that allows for optimal parameter selection prior to carrying out a numerical simulation. In what follows, a linear stability analysis framework is developed in which the relationship between LBM parameters, such as the time step and relaxation time, and flow regime parameters such as the Reynolds and Mach numbers will be exploited. The analysis presented here differs from existing studies [16-18] as it is formulated specifically for the single-population method with arbitrary specific heat ratio in section III. Additionally it accepts input parameters of Mach and Reynolds number, which is particularly useful to aerospace problems. It is applicable to any general flow problem with a characteristic Mach and Reynolds number, and shall be applied in later sections to aid the choice of time step for numerical simulations of the nozzle test case. Although not covered in this work, this methodology is also applicable to other discretizations and collision operators. There are limitations to this analysis, mainly that the effects of boundary conditions are not accounted for. Nonlinear stability, related to the ability to capture discontinuities in the solution is also not studied in this work

Accepted manuscript: AIAA SciTech 2021 Forum, https://doi.org/10.2514/6.2021-1663

Copyright (c) 2021 by the American Institute of Aeronautics and Astronautics, Inc. All rights reserved. 


\section{A. Linearization of the Discrete Velocity Boltzmann Equation}

Before the stability analysis can be applied, a linear estimate of the collision operator must be derived, leading to a linearized form of the DVBE. The particle distributions may be represented in a concise vector form, where the vectors $\boldsymbol{f}=\left[f_{0}, f_{1}, \ldots, f_{N-1}\right]^{T}$ and $\boldsymbol{\xi}=\left[\xi_{0}, \xi_{1}, \ldots, \xi_{N-1}\right]^{T}$ contain the particle distributions and discrete velocities respectively. The collision operator is also written as a vector-valued function $\boldsymbol{\Omega}(\mathbf{f})$, which is assumed to be nonlinear. Partial derivatives in space and time shall now be written more concisely as $\partial_{t}$ and $\partial_{x}$, and $\boldsymbol{I}$ represents the $N \times N$ identity matrix. The DVBE may now be written as the matrix system shown in Eq. (17).

$$
\partial_{t} \boldsymbol{f}+\boldsymbol{I} \xi \partial_{x} \boldsymbol{f}=\boldsymbol{\Omega}(\boldsymbol{f})
$$

Following the method of Siebert et al. [18], the linearization is performed around a base flow at equilibrium $f^{e q}$ given by a specified density, velocity and temperature. A first-order perturbation $\delta \boldsymbol{f}$ in the particle distribution is imposed, which gives a total particle distribution function of $f=f^{e q}+\delta \boldsymbol{f}$. A linear approximation of the collision operator at the perturbed state may be derived by taking a forward Taylor expansion about the equilibrium, as shown in Eq. [18). Similarly to existing studies [17, 18], the first order approximations to the particle distribution function and collision operator are then substituted into Eq. (17) to give the linearized DVBE shown in Eq. (19). This equation governs the time evolution of the perturbation.

$$
\begin{gathered}
\boldsymbol{\Omega}\left(\boldsymbol{f}^{e q}+\delta \boldsymbol{f}\right)=\boldsymbol{\Omega}\left(\boldsymbol{f}^{e q}\right)+\left.\frac{\partial \boldsymbol{\Omega}}{\partial \boldsymbol{f}}\right|_{\boldsymbol{f}=\boldsymbol{f}^{e q}} \delta \boldsymbol{f}+O\left(\delta \boldsymbol{f}^{2}\right) \\
\partial_{t} \delta \boldsymbol{f}+\boldsymbol{I} \boldsymbol{\xi} \partial_{x} \delta \boldsymbol{f}=\left.\frac{\partial \boldsymbol{\Omega}}{\partial \boldsymbol{f}}\right|_{\boldsymbol{f}=\boldsymbol{f}} \delta \boldsymbol{f}
\end{gathered}
$$

The collision operator is now specified as the SRT-BGK, which is shown in vector form in Eq. 20p. Its Jacobian with respect to $f$ is shown in Eq. 21]. For the above LB method, the Jacobian of $\mathbf{f}^{\mathbf{e q}}$ with respect to $\mathbf{f}$ can be derived using the moments equation (8). The chosen methodology for this is described in the Appendix, and from here onwards it shall be assumed that the value of this Jacobian may be obtained from fluid quantities $\rho, u, \theta$. The linearized DVBE with the BGK collision operator shown in Eq. 222) is obtained by substitution of Eq. 21) into Eq. 19].

$$
\begin{aligned}
\mathbf{\Omega}(\boldsymbol{f}) & =-\frac{1}{\tau}\left(\boldsymbol{f}-\boldsymbol{f}^{e q}\right) \\
\frac{\partial \boldsymbol{\Omega}(\boldsymbol{f})}{\partial \boldsymbol{f}} & =-\frac{1}{\tau}\left(\boldsymbol{I}-\frac{\partial \boldsymbol{f}^{e q}}{\partial \boldsymbol{f}}\right) \\
\partial_{t} \delta \boldsymbol{f}+\boldsymbol{I} \boldsymbol{\xi} \partial_{x} \delta \boldsymbol{f} & =-\frac{1}{\tau}\left(\boldsymbol{I}-\left.\frac{\partial \boldsymbol{f}^{e q}}{\partial \boldsymbol{f}}\right|_{\boldsymbol{f}=\boldsymbol{f}^{e q}}\right) \delta \boldsymbol{f}
\end{aligned}
$$

\section{B. Von-Neumann Analysis of the Linearized Boltzmann Equation}

The Von Neumann technique is used for the estimation of linear stability of a numerical scheme, by studying the behavior of wave perturbations in the frequency spectrum. A non-dimensional discrete spatial domain of length $2 \pi$ is defined, on which the solution $f_{i}^{n}$ is captured. A Fourier transform of the solution is performed, so that it is now represented as a sum of harmonics $F_{k}^{n} e^{I k x_{i}}$. The wave numbers are specified as integer values $k=1,2, \ldots, k_{\max }$, where $k_{\max }$ corresponds to highest frequency harmonic of the solution, and is chosen to give a sufficient resolution of the frequency spectrum. The above series of $k$ covers the range $k \Delta x=(0,2 \pi)$, thus considering all possible harmonics to be captured within the domain. The Von Neumann stability criterion is defined by the amplification factor $F_{k}^{n+1} / F_{k}^{n}$, which represents the growth of a harmonic with wave number $k$ between two consecutive time intervals. This must be satisfied for all harmonics with the above wave numbers. Next, Eq. 22] is discretized using the Beam-Warming method similarly to Eqs. 90 - $(10)$, followed by a Fourier transform to give Eqs. $237-(24)$, where $F_{\alpha, k}^{n}$ represents the amplitude component of a harmonic belonging to the spatial solution of population $f_{\alpha}$. The linear approximation for the BGK collision operator has been included.

$$
\begin{array}{r}
F_{\alpha, k}^{n+1}=F_{\alpha, k}^{n}+\frac{1}{2} \xi_{\alpha} \frac{\kappa}{|\xi|_{\max }}\left(3 F_{\alpha, k}^{n}-4 F_{\alpha, k}^{n} e^{I k \Delta x}+F_{\alpha, k}^{n} e^{I 2 k \Delta x}\right) \\
+\frac{1}{2}\left(\xi_{\alpha} \frac{\kappa}{|\xi|_{\max }}\right)^{2}\left(F_{\alpha, k}^{n}-2 F_{\alpha, k}^{n} e^{I k \Delta x}+F_{\alpha, k}^{n} e^{I 2 k \Delta x}\right)+\left.\Delta t \frac{\partial \Omega_{\alpha}}{\partial f_{\beta}}\right|_{f=f e q} F_{\alpha, k}^{n}
\end{array}
$$

Accepted manuscript: AIAA SciTech 2021 Forum, https://doi.org/10.2514/6.2021-1663

Copyright (c) 2021 by the American Institute of Aeronautics and Astronautics, Inc. All rights reserved. 


$$
\begin{array}{r}
F_{\alpha, k}^{n+1}=F_{\alpha, k}^{n}-\frac{1}{2} \xi_{\alpha} \frac{\kappa}{|\xi|_{\max }}\left(3 F_{\alpha, k}^{n}-4 F_{\alpha, k}^{n} e^{-I k \Delta x}+F_{\alpha, k}^{n} e^{-2 I k \Delta x}\right) \\
+\frac{1}{2}\left(\xi_{\alpha} \frac{\kappa}{|\xi|_{\max }}\right)^{2}\left(F_{\alpha, k}^{n}-2 F_{\alpha, k}^{n} e^{-I k \Delta x}+F_{\alpha, k}^{n} e^{-I 2 k \Delta x}\right)+\left.\Delta t \frac{\partial \Omega_{\alpha}}{\partial f_{\beta}}\right|_{f=f e q} F_{\alpha, k}^{n}
\end{array}
$$

Equations 23 - 24 are next written as a matrix system $\boldsymbol{F}^{n+1}=\boldsymbol{L} \boldsymbol{F}^{n}$, with $\boldsymbol{L}$ given by Eq. 25. The matrix $\boldsymbol{\Xi}=\boldsymbol{I} \boldsymbol{\xi}$ is defined, which is a diagonal matrix containing the discrete velocities. The linearized BGK collision operator has now been included. The diagonal matrices $\boldsymbol{E}_{\mathbf{1}}$ and $\boldsymbol{E}_{\mathbf{2}}$ contain the spatial discretization components of the equation. For $\alpha=0,1, \ldots, 3$ and $\beta=0,1, \ldots 3, E_{1_{\alpha \beta}}=\left(-3+4 e^{I k \Delta x}-e^{I 2 k \Delta x}\right) \delta_{\alpha \beta}$ and $E_{2_{\alpha \beta}}=\left(1-2 e^{I k \Delta x}+e^{I 2 k \Delta x}\right) \delta_{\alpha \beta}$. For $\alpha=4,5,6$ and $\beta=4,5,6, E_{1_{\alpha \beta}}=\left(3-4 e^{-I k \Delta x}+e^{-I 2 k \Delta x}\right) \delta_{\alpha \beta}$ and $E_{2_{\alpha \beta}}=\left(1-2 e^{-I k \Delta x}+e^{-I 2 k \Delta x}\right) \delta_{\alpha \beta}$. The amplification factors of the system are given by the eigenvalues of $\boldsymbol{L}$, meaning the magnitudes of all seven eigenvalues must remain below one for stability. The eigenvalues are computed numerically, using the Python Numpy library.

$$
\boldsymbol{L}=\boldsymbol{I}-\frac{1}{2} \frac{\kappa}{\left|\xi_{\text {max }}\right|} \boldsymbol{\Xi} \boldsymbol{E}_{1}+\frac{1}{2} \frac{\kappa^{2}}{\left|\xi_{\text {max }}\right|^{2}} \boldsymbol{\Xi} \boldsymbol{\Xi} \boldsymbol{E}_{2}-\frac{\Delta t}{\tau}\left(\boldsymbol{I}-\left.\frac{\partial \boldsymbol{f}^{\boldsymbol{e q}}}{\partial \boldsymbol{f}}\right|_{\boldsymbol{f}=\boldsymbol{f} \boldsymbol{e q}}\right)
$$

\section{Expression of Collision Operator in Terms of Flow Regime}

Since $\tau$ is often the parameter chosen in practice to achieve stability, it is common to study the stability of LB methods with $\tau$ or $\omega=\Delta t / \tau$ as the independent variable. These parameters appear naturally in the LBM formulation, but they are not intelligible in terms of a real world configuration. Thus, we reveal the connection between them and the parameters that characterize a flow regime, shedding light on the consequences of certain parameter choices and informing on the needs of a generic computation. Specifically, the chosen parameters are fluid Mach number $(M)$ and grid size based Reynolds number $\left(R e_{g}\right)$, which are defined by Eqs. (26) and (27). As mentioned previously, the values for static quantities $\rho, u, \theta$ are also required to specify the Jacobian of $\boldsymbol{f}^{\boldsymbol{e} q}$, meaning that a reference temperature $T$ must also be specified. The relationship $\mu=\tau \rho \theta$ between dynamic viscosity and relaxation time, and the previously defined advective Courant number are also used. The $\Delta t / \tau$ component of the time-integrated collision operator in Eq. (25) can then be substituted for a function of the above fluid properties. This is shown in Eq. [28), which shall be used to generate the stability maps in section $\mathrm{VI}$.

$$
\begin{gathered}
M=\frac{u}{\sqrt{\gamma \theta}} \\
R e_{g}=\frac{\rho u \Delta x}{\mu} \\
\boldsymbol{L}=\boldsymbol{I}-\frac{1}{2} \frac{\kappa}{\left|\xi_{\text {max }}\right|} \boldsymbol{\Xi} \boldsymbol{E}_{\mathbf{1}}+\frac{1}{2} \frac{\kappa^{2}}{\left|\xi_{\text {max }}\right|^{2}} \boldsymbol{\Xi} \boldsymbol{\Xi} \boldsymbol{E}_{\mathbf{2}}-\frac{\kappa \operatorname{Re}_{g} \sqrt{\gamma \theta}}{M \gamma\left|\xi_{\text {max }}\right|}\left(\boldsymbol{I}-\left.\frac{\partial \boldsymbol{f}^{\boldsymbol{e q}}}{\partial \boldsymbol{f}}\right|_{\boldsymbol{f}=\boldsymbol{f}}\right)
\end{gathered}
$$

\section{Numerical Simulations}

\section{A. One-Dimensional Shock Tube}

The Sod's shock tube test case [19] enables validation of the LB method to capture discontinuous solutions. The domain of length 1 spans $x=-0.5$ to $x=0.5$, with the discontinuity located initially at $x=0$. Initial conditions of $\left(\rho_{L}, u_{L}, p_{L}\right)=(1,0,1)$ and $\left(\rho_{R}, u_{R}, p_{R}\right)=(0.125,0,0.1)$ were applied to the left and right sections respectively. In this instance, the domain was divided into $N=4000$ intervals, giving a grid size of $\Delta x=2.5 \times 10^{-4}$. Linear stability analysis cannot be used to decide the time step for this case, as the solution is discontinuous. Instead it was found through experiment that a Courant number of $\kappa=0.2$ provided a stable solution, giving a time step of $\Delta t=1.67 \times 10^{-5}$. Relaxation time was chosen based on an $\omega$ value of 0.8 , leading to $\tau=2.09 \times 10^{-5}$. The observed stability limit is considerably low compared to existing studies with similar methods. In the work of Dellar [9], for which the Beam-Warming scheme was also used, but with the collision operator included through a Strang-split procedure, stable shock tube solution was achievable at a higher $\kappa$ of 0.9 . This suggests that by including the collision operator directly

Accepted manuscript: AIAA SciTech 2021 Forum, https://doi.org/10.2514/6.2021-1663

Copyright @ 2021 by the American Institute of Aeronautics and Astronautics, Inc. All rights reserved. 
into the Beam-Warming scheme, the maximum stable time step has reduced significantly, and that stability is sensitive to the way that the collision operator is is discretized.

Figure 1 shows that when compared with the exact solution obtained from a Riemann solver, the LB method shows high accuracy with regard to instantaneous shock strength and position, and the rarefaction wave is captured very well. The main areas of inaccuracy are the overshoots and oscillations around the shock. These were found to be somewhat alleviated with an increased fluid viscosity from a larger relaxation time. To further correct this discrepancy would require modification of the solution scheme, for example the inclusion of a limiter. The contact discontinuity does not feature such overshoots, although is smoothed.

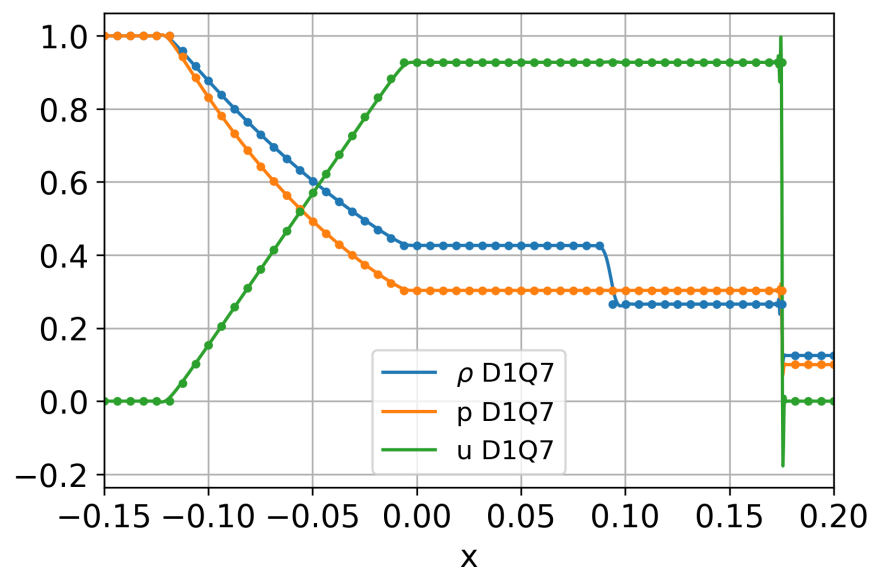

Fig. 1 Sod shock tube solution given by LBM (solid) and exact (dotted), at $\mathrm{t}=0.1$

\section{B. Convergent-Divergent Nozzle}

The convergent-divergent nozzle test case is a steady-state case effective for validating the accuracy of onedimensional compressible flow solvers. As shown in Eq. [14], the LB method is made suitable to model this case through the addition of a source term to the DVBE. The flow is assumed to be inviscid, isentropic and uniform in the cross sectional plane, which enables a reduction of the problem from three-dimensional to one-dimensional space. There exists an exact solution given by the isentropic nozzle flow equations, which for this study was obtained using a root-finding algorithm. The exact solution is used to quantify the error of the LB method.

Figure 2 shows the convergent-divergent nozzle of length $L=1 \mathrm{~m}$, with the centreline parallel to the $\mathrm{x}$ axis, and the throat positioned at $x=0.5 \mathrm{~m}$. The cross sectional area at both the inlet and outlet is $A_{\text {in }}=0.15 \mathrm{~m}^{2}$, and the minimum value is $A_{t}=0.1 \mathrm{~m}^{2}$ at the throat. The area distribution between follows a cosine function $A(x)=a \cos (2 \pi x)+b$, where $a=0.5\left(A_{\text {in }}-A_{t}\right)$ and $b=0.5\left(A_{\text {in }}+A_{t}\right)$. The flow through the nozzle is constrained with Dirichlet boundary conditions of total pressure $p_{0}$ and total temperature $T_{0}$ at the inlet, and static pressure $p_{\text {out }}$ at the outlet. For this study, the boundary conditions were defined with physical units; $p_{0}=1.01 \times 10^{5} \mathrm{~Pa}$ and $T_{0}=291 \mathrm{~K}$. The chosen outlet pressure $p_{\text {out }}=9.09 \times 10^{4}$ maintains a subsonic flow, which ensures a smooth solution. A grid size $\Delta x=0.01 \mathrm{~m}$ was chosen for the initial cases.

Accepted manuscript: AIAA SciTech 2021 Forum, https://doi.org/10.2514/6.2021-1663

Copyright (C) 2021 by the American Institute of Aeronautics and Astronautics, Inc. All rights reserved. 


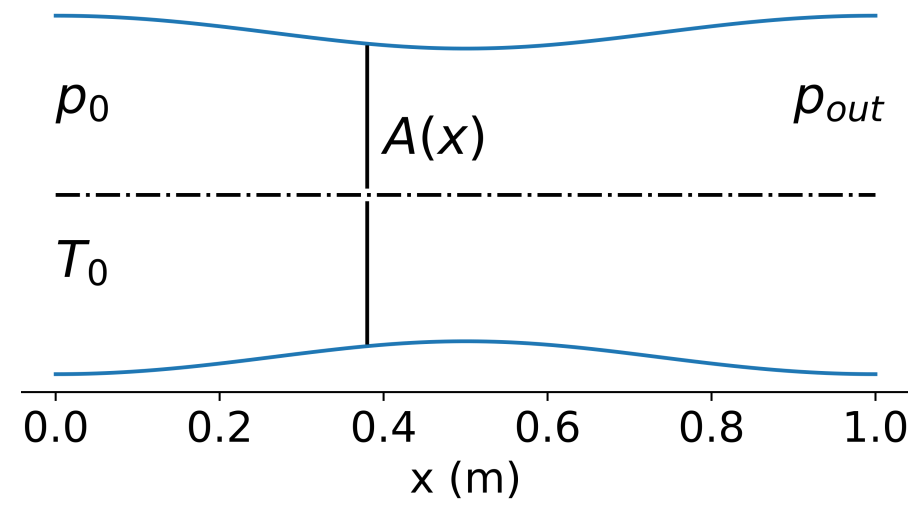

Fig. 2 Diagram of nozzle geometry and boundary conditions

\section{Stability Analysis}

Using the methodology of section $\mathrm{V}$ stability maps were generated to show for which values of $M, R e_{g}$ and $\kappa$ a linearly stable solution is predicted by Eq. (28). These may also be used to aid the choice of $\kappa$ and $R e_{g}$ to be used in the numerical experiment. The exact solution to the nozzle case represents an inviscid flow, meaning that the grid Reynolds number of the simulation should be as high as possible to minimise errors due to fluid viscosity. Since it is the steady state solution that is required, it is desirable to lower computational cost by choosing the largest possible time step within the stability limit, meaning $\kappa$ should be as large as possible.

Equation (28) requires inputs of $(M, R e, \theta, \kappa)$, which could be given by any general flow problem. For this study, they are specified by the considered example of converging-diverging nozzle flow with an inlet Mach number of 0.39 and throat Mach number of 0.72 . Additionally at the inlet and throat, the strength of the source term $S$ is zero because $d A / d x=0$, therefore the effect of the source term on stability was not considered during this study. Future work should include analysis of source terms, which may reveal additional stability limitations for this case. Next, $\kappa$ and $R e_{g}$ were chosen to be the independent variables for the stability map. As can be seen in Eq. 27,, varying $R e_{g}$ is analogous to varying the fluid viscosity, which can be controlled by the relaxation time. Additionally, the Jacobian within the collision operator requires inputs of $(\rho, u, \theta)$, which for this case can be calculated using the nozzle boundary conditions $p_{0}, T_{0}$, and the above $M$ values, by using the isentropic flow equations.

The shaded areas of Fig. 3 represent the range of $R e_{g}$ and $\kappa$ for which all eigenvalues of the stability matrix in Eq. (24) are less than one for all harmonics of $k=1,2, \ldots, k_{\max }$. In this case $k_{\max }=10$ was chosen to give sufficient resolution. It is observed that a lower $\kappa$ generally results in a wider range of stable $R e_{g}$, and as $\kappa$ is increased, the maximum stable $R e_{g}$ decreases drastically. From Fig. 3a it is estimated that the maximum linearly stable $\kappa$ value for this case is 0.25 , and is limited by the condition of lowest Mach number. It is apparent that a more favourable stability region is found at the higher Mach number, where for a given $R e_{g}$, the maximum stable $\kappa$ is larger. This figure shows also that at Mach 0.39 there is estimated to be a lower limit for grid Reynolds number of approximately five, meaning that an upper limit exists for fluid viscosity, which varies with Mach number.

The observed $\kappa$ limits are unexpectedly low in comparison with LB methods which use the collision-streaming scheme, for which a fixed $\kappa=1$ is imposed. The reason for such limited stability remains uncertain, although it is suspected that one contribution is due to the introduction of fixed internal energy coefficients by the method of Kataoka and Tsutahara [6]. The analysis of Siebert et al. [18] shows that single-population LB methods for compressible flow which use the collision-streaming scheme, without the use of such internal energy coefficients, may achieve stability for smooth solutions. These methods however are limited to a fixed arbitrary specific heat ratio. Similarly to the shock tube, it may be that an improvement in stability would be seen if the collision operator were included using the Strang-split approach.

Accepted manuscript: AIAA SciTech 2021 Forum, https://doi.org/10.2514/6.2021-1663

Copyright (c) 2021 by the American Institute of Aeronautics and Astronautics, Inc. All rights reserved. 


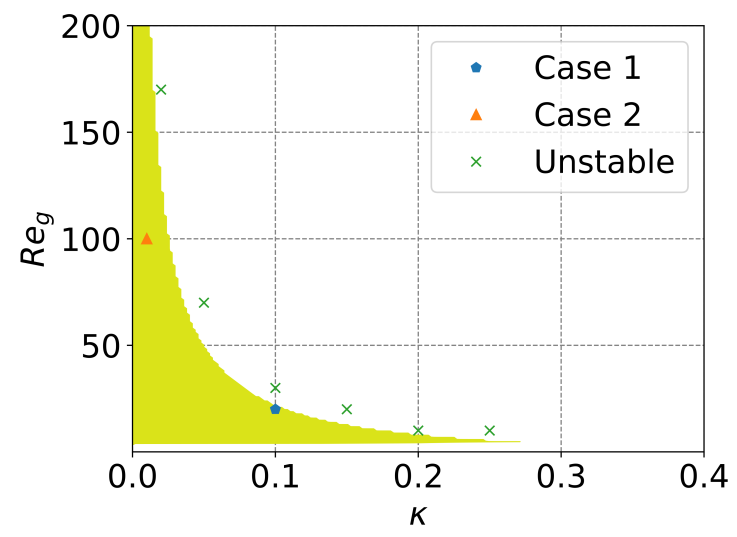

(a) Inlet: $M=0.39$

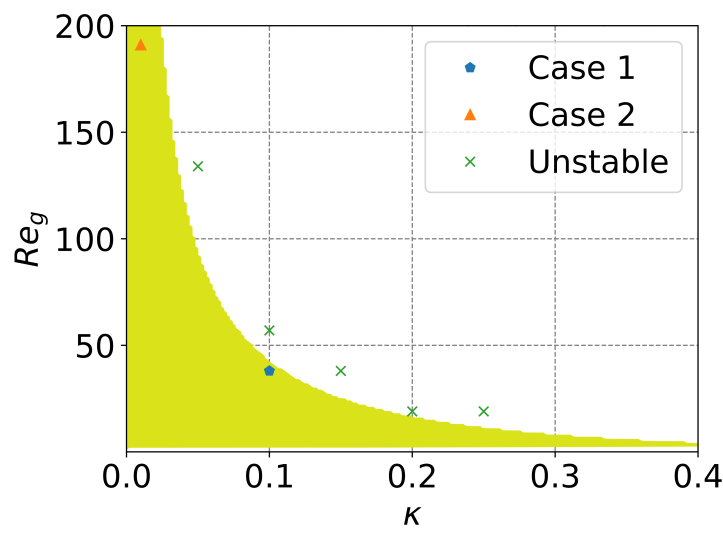

(b) Throat: $M=0.72$

Fig. $3 R e_{g}$ vs. $\kappa$ stability maps for the conditions of the nozzle test case

Numerical simulations were performed at two different values of $R e_{g}$, so that the effect of fluid viscosity on error could be examined. The stability maps were used to guide the choice of $\kappa$ and $R e_{g}$, however it is not clear beforehand whether the inlet or throat conditions are the most restrictive to stability. It was therefore decided to begin at the inlet, and then check at the throat. Firstly, two simulation cases were defined by choosing $\left(\kappa, R e_{g_{\text {inlet }}}\right)=(0.1,20)$ and $(0.01,100)$ respectively, which as shown in Fig. $3 \mathrm{a}$. are within the stability region of the inlet. These values of $R e_{g_{\text {inlet }}}$ were then used with Eq. 27) and $\mu=\tau \rho \theta$ to calculate $\tau$ for each case. It was then possible to use the values of $\tau$ to calculate the $R e_{g}$ at the throat conditions, which are 38.3 and 191 for the first and second case respectively. Figure $3 \mathrm{~b}$ was then used to check that the cases are also stable at the throat. The parameters for each case are summarized in Table 1 In addition, numerical simulations were performed at a series of conditions outside of the stability region. These are also shown in Fig. 3 , and were all found to be unstable in practice, thus demonstrating the accuracy of the analysis.

\section{Table 1 Summary of parameters for nozzle simulation cases}

\begin{tabular}{lll}
\hline \hline Parameter & Case 1 & Case 2 \\
\hline$R e_{g_{\text {inlet }}}$ & 20 & 100 \\
$R e_{g_{\text {throat }}}$ & 38.3 & 191 \\
$\kappa$ & 0.1 & 0.01 \\
$p_{0}, \mathrm{~Pa}$ & $1.01 \times 10^{5}$ & $1.01 \times 10^{5}$ \\
$T_{0}, \mathrm{~K}$ & 291 & 291 \\
$p_{\text {out }}, \mathrm{Pa}$ & $9.09 \times 10^{4}$ & $9.09 \times 10^{4}$ \\
$\Delta x, \mathrm{~m}$ & 0.01 & 0.01 \\
$\mu_{\text {ref }}$, Pa.s & $7.54 \times 10^{-3}$ & $1.51 \times 10^{-3}$ \\
$\tau, s$ & $8.31 \times 10^{-8}$ & $1.66 \times 10^{-8}$ \\
\hline \hline
\end{tabular}

In order to estimate the range of Mach numbers for which stability is predicted for each case, stability maps of $R e_{g}$ against Mach number were created. These are presented in Fig. 4, and show that for both cases there exists a stable region for all subsonic Mach numbers. The points representing the inlet and throat conditions are included. The lower $K$ value of the second case results in a significantly larger stability region. For both cases, the maximum stable grid Reynolds number increases with Mach number, and the lower limit of grid Reynolds number varies nonlinearly with Mach number.

Accepted manuscript: AIAA SciTech 2021 Forum, https://doi.org/10.2514/6.2021-1663

Copyright (c) 2021 by the American Institute of Aeronautics and Astronautics, Inc. All rights reserved. 


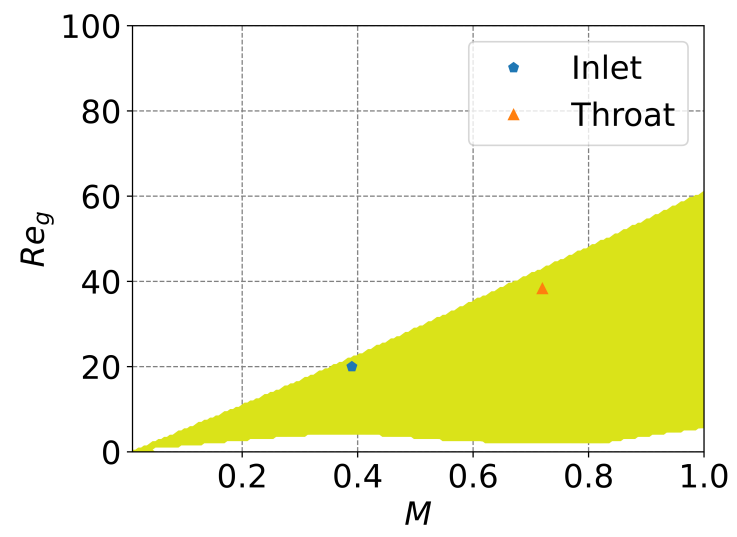

(a) Case 1: $\kappa=0.1$

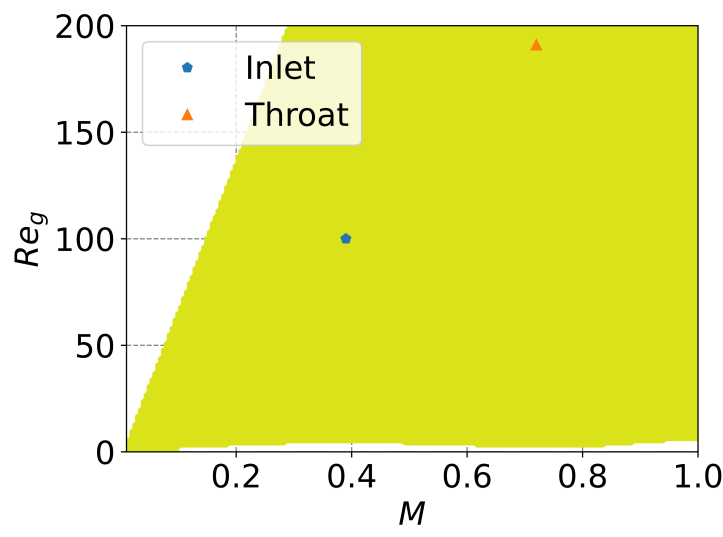

(b) Case 2: $\kappa=0.01$

Fig. $4 R e_{g}$ vs. $M$ stability maps for both nozzle test cases

\section{Simulation Results}

Figure 5 shows the solutions to the two nozzle test cases described in Table 1, for which the choice of $\kappa$ and $R e_{g}$ were guided by the preceding stability analysis. The non-equilibrium extrapolation method [25] was used to prescribe the boundary values for the $f_{\alpha}$ populations. As the nozzle is a steady-state case, a convergence criteria is required to determine at which time to take the solution. For this study, this is once the average relative change in all macroscopic quantities is no larger than a tolerance of $10^{-12}$. Such a strict tolerance is manageable in terms of cost for one-dimensional validation cases such as this. The LBM solutions contain a small amount of error compared with the exact solution, in the form of a slight underestimation of Mach number. This is especially noticeable at the throat. Using the root mean squared (RMS) method shown in Eq. 29p, the average error in Mach number of the first case is $2.97 \times 10^{-3}$. For a fixed grid size and boundary conditions, the error may be reduced by increasing the grid Reynolds number, resulting in a lower fluid viscosity. This is shown by the solution to the second case, for which the RMS error is a lower value of $7.12 \times 10^{-4}$. This improved accuracy has however been achieved at the cost of increased computational expense, given the stricter $\kappa$ limit.

$$
e_{R M S}=\sqrt{\sum_{i=1}^{N_{x}} \frac{\left(M_{i_{L B M}}-M_{i_{\text {exact }}}\right)}{N_{x}}}
$$




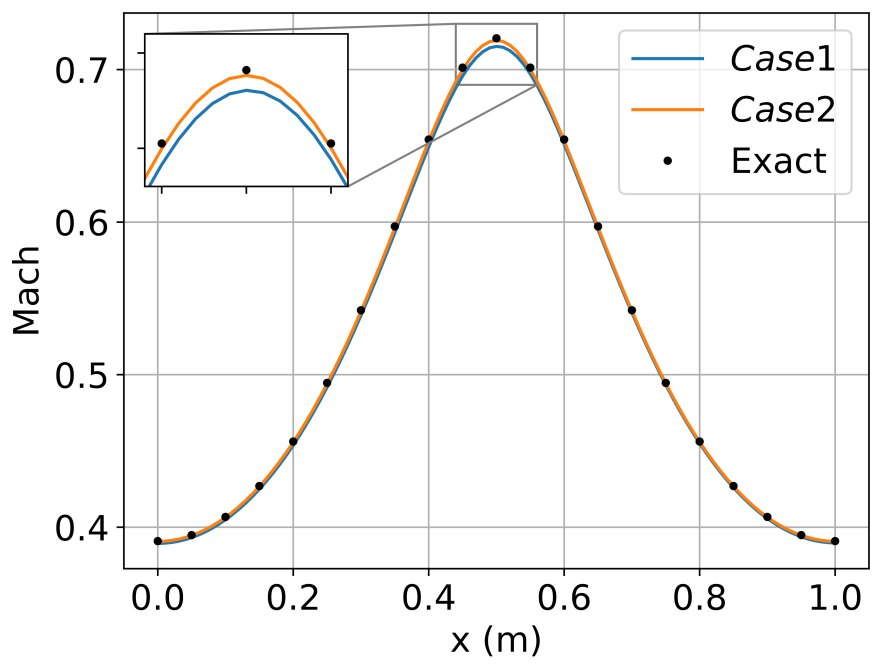

Fig. 5 Mach number solution of quasi one-dimensional nozzle

Next the dependency of error on grid size was studied by further simulations of the nozzle test case. Error in Mach number is again quantified by the RMS error against the exact solution. The same area profile and boundary conditions as for the solution shown in Fig. 5]were used. Simulations were performed at two different values of reference fluid viscosity. It can be seen in Fig. 6 that for larger values of $\Delta x$, the gradients of both curves agree well with the theoretical second order of convergence. The error here consists mostly of discretization error, and the difference between both sets of simulations is not noticeable. At smaller values of $\Delta x$, the lower discretization error means that the error due to fluid viscosity becomes comparatively more significant, and so the error decreases at a lower rate. This is noticeably greater for the simulations of larger fluid viscosity. As $\Delta x \rightarrow 0$ the error will reach an asymptotic value determined by the error due to viscosity.

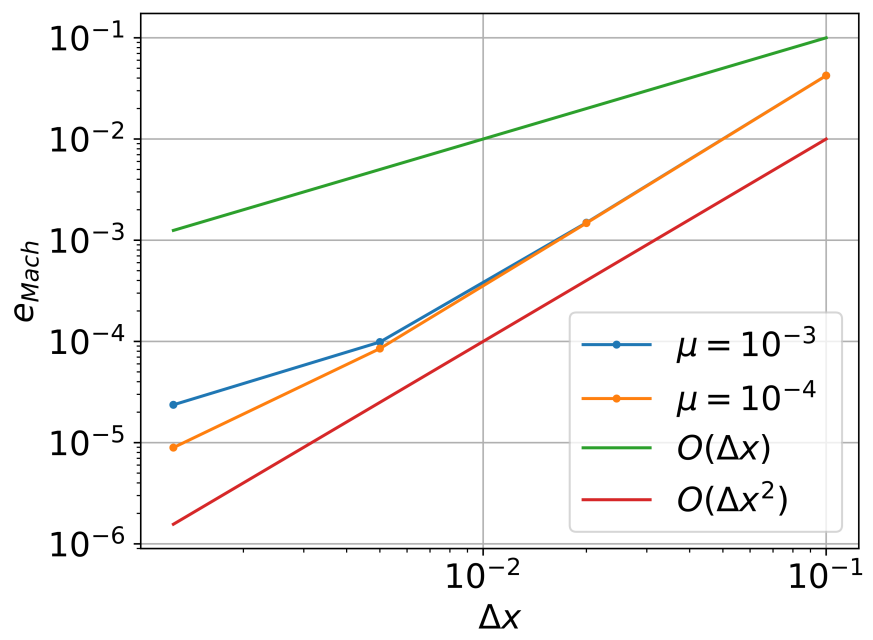

Fig. 6 Average error in Mach number vs. grid size

\section{Conclusion}

The stability and accuracy of the single-population LBM with arbitrary specific heat ratio was evaluated using theoretical analysis and numerical simulations. A linear stability analysis based on the von Neumann method was presented, which may be used to estimate the linear stability of a general flow problem defined by a characteristic Mach and Reynolds number. The analysis was applied to obtain stability maps, which showed that in general a lower advective 
Courant number shall enable a higher Reynolds number to be achieved. The absolute limits on advective Courant number were considerably low, which imposed strict limits on the choice of time step size for numerical simulations. For a fixed advective Courant number, it was found that stability was achievable for the entire subsonic regime, and that a higher Mach number enabled a higher grid Reynolds number. In addition, a lower limit for grid Reynolds number was found, which varied nonlinearly with Mach number.

The accuracy of the LB method was studied using two Euler test cases. Shock tube simulations highlighted the effectiveness of the finite difference space-time scheme for stably resolving discontinuities. The leading source of error was the overshoots and oscillations near the shock. The quasi one-dimensional nozzle test case enabled the evaluation of accuracy for a smooth solution, over a range of Mach numbers. The preceding stability analysis provided guidance for the choice of advective Courant number to be used, and showed that stability would be maintained across the Mach number range covered by the case. Solutions for macroscopic properties were obtained with high accuracy, although there was a small error source due to the non-zero fluid viscosity introduced by the collision operator. The order of convergence of error with respect to grid size agreed closely to the theoretical second order of the scheme, although does not tend to zero in the fine grid size limit due to the viscosity error.

The observed accuracy from both test cases is promising toward the future goal of extension to multiple dimensions and modelling of a wider range of engineering flow cases. The stability limitation on the time step however presents concerns with regards to the computational expense. Future work will begin by identifying the attributes of the current method which cause these limits. The study and comparison of other LB methods for compressible flow, such as the two-population method, should prove beneficial towards identifying any potential improvements to be gained by modification of the current approach.

\section{Appendix: Jacobian of Equilibrium Distribution}

The following methodology covers the derivation of the Jacobian of the equilibrium distribution function vector $\boldsymbol{f}^{\boldsymbol{e q}}$ with respect to the particle distribution function vector $f$. In general the equilibrium distributions $f^{e q}$ are nonlinear functions of macroscopic quantities, which are themselves nonlinear functions of the particle distributions. To begin, define a vector $\boldsymbol{y}$ of macroscopic quantities which we shall refer to as primitive quantities:

$$
\boldsymbol{y}(\boldsymbol{f})=\left[\begin{array}{l}
\rho(\boldsymbol{f}) \\
u(\boldsymbol{f}) \\
\theta(\boldsymbol{f})
\end{array}\right]
$$

Through the definitions of the particle distribution moments, and the use of the D1Q7 discretization, the primitive variables are nonlinear functions of $\boldsymbol{f}$, although deriving these functions would be comparatively complicated. It is easier instead to consider that the same moment definitions provide linear functions in $\boldsymbol{f}$, for conservative macroscopic quantities $\boldsymbol{w}$. These conservative quantities can therefore either be expressed in terms of primitive macroscopic variables, or distribution functions, as shown respectively:

$$
\begin{gathered}
\boldsymbol{w}(\boldsymbol{y})=\left[\begin{array}{c}
\rho \\
\rho u \\
\rho u^{2}+2 \frac{\rho \theta}{(\gamma-1)}
\end{array}\right] \\
\boldsymbol{w}(\boldsymbol{f})=\left[\begin{array}{ccccccc}
1 & 1 & 1 & 1 & 1 & 1 & 1 \\
-3 & -2 & -1 & 0 & 1 & 2 & 3 \\
9 & 4 & 2 & 4 & 2 & 4 & 9
\end{array}\right]\left[\begin{array}{l}
f_{0} \\
f_{1} \\
f_{2} \\
f_{3} \\
f_{4} \\
f_{5} \\
f_{6}
\end{array}\right]
\end{gathered}
$$

Next, the definition of the equilibrium distributions provided by the D1Q7 discretization is used. This comes from the system $\boldsymbol{C} \boldsymbol{f}^{e q}=\boldsymbol{m}$, shown fully in Eq. (8). The equilibrium distribution function can then be expressed as a function of $\boldsymbol{f}$, as in Eq. 33. The application of the matrix chain rule then leads to the required Jacobian Eq. (34).

Accepted manuscript: AIAA SciTech 2021 Forum, https://doi.org/10.2514/6.2021-1663

Copyright (C) 2021 by the American Institute of Aeronautics and Astronautics, Inc. All rights reserved. 


$$
\begin{gathered}
f^{e q}=C^{-1} m(y(w(f))) \\
\frac{\partial f^{e q}}{\partial f}=C^{-1} \frac{\partial m}{\partial y} \frac{\partial y}{\partial w} \frac{\partial w}{\partial f}
\end{gathered}
$$

Next the three Jacobian matrices on the right hand side of Eq. (34) must be derived. The right hand side of Eq. (8) gives $\boldsymbol{m}(\boldsymbol{y})$. Taking the Jacobian of this function gives Eq. 35.

$$
\frac{\partial \boldsymbol{m}}{\partial \boldsymbol{y}}=\left[\begin{array}{ccc}
1 & 0 & 0 \\
u & \rho & 0 \\
u^{2}+\theta & 2 \rho u & \rho \\
u^{3}+3 u \theta & 3 \rho u^{2}+3 \rho \theta & 3 \rho u \\
u^{2}+\frac{2}{\gamma-1} \theta & 2 \rho u & \frac{2}{\gamma-1} \rho \\
u^{3}+\frac{2 \gamma}{\gamma-1} u \theta & 3 \rho u^{2}+\frac{2 \gamma}{\gamma-1} \rho \theta & \frac{2 \gamma}{\gamma-1} \rho u \\
u^{4}+\frac{5 \gamma-3}{\gamma-1} u^{2} \theta+\frac{2 \gamma}{\gamma-1} \theta^{2} & 4 \rho u^{3}+2 \frac{5 \gamma-3}{\gamma-1} \rho u \theta & \frac{5 \gamma-3}{\gamma-1} \rho u^{2}+\frac{4 \gamma}{\gamma-1} \rho \theta
\end{array}\right]
$$

Next is to derive the Jacobian of the primitive variables with respect to the conservative variables. First express the primitive variables as a function of the conserved variables. For this, adopt the notation $\boldsymbol{w}(\boldsymbol{y})=\left[\begin{array}{lll}w_{1} & w_{2} & w_{3}\end{array}\right]^{T}$, with $w_{1}=\rho, w_{2}=\rho u$, and $w_{3}=\rho u^{2}+2 \frac{\rho \theta}{(\gamma-1)}$, to simplify the previously expressed form in Eq. 31p. Using this with Eq. (30) gives Eq. 36. Taking the Jacobian of Eq. (36) gives the required Jacobian Eq. 37.

$$
\begin{aligned}
& \boldsymbol{y}(\boldsymbol{w})=\left[\begin{array}{c}
w_{1} \\
\frac{w_{2}}{w_{1}} \\
(\gamma-1) \frac{1}{2 w_{1}}\left(w_{3}-\frac{w_{2}^{2}}{w_{1}}\right)
\end{array}\right] \\
& \frac{\partial \boldsymbol{y}}{\partial \boldsymbol{w}}=\left[\begin{array}{ccc}
1 & 0 & 0 \\
\frac{-w_{2}}{w_{1}^{2}} & \frac{1}{w_{1}} & 0 \\
-(\gamma-1) \frac{w_{3}}{2 w_{1}^{2}}+(\gamma-1) \frac{w_{2}^{2}}{w_{1}^{3}} & -(\gamma-1) \frac{w_{2}}{w_{1}^{2}} & (\gamma-1) \frac{1}{2 w_{1}}
\end{array}\right]
\end{aligned}
$$

The last required Jacobian, shown in Eq. 38), is of the conserved variables with respect to the distribution functions. This is provided by the moment equations for density, momentum, and total energy, which can be seen in Eq. (8).

$$
\frac{\partial \boldsymbol{w}}{\partial \boldsymbol{f}}=\left[\begin{array}{ccccccc}
1 & 1 & 1 & 1 & 1 & 1 & 1 \\
-3 & -2 & -1 & 0 & 1 & 2 & 3 \\
9 & 4 & 2 & 4 & 2 & 4 & 9
\end{array}\right]
$$

Now that every required matrix has been derived, we may multiply each as shown in Eq. (34) to obtain the equilibrium distribution Jacobian. Numerical values of this Jacobian are obtained by specifying inputs of macroscopic flow quantities $\rho, u, \theta$, as these are required to specify $\frac{\partial m}{\partial y}$ and $\frac{\partial y}{\partial w}$.

\section{Acknowledgments}

The authors thank Rolls-Royce plc and the EPSRC for the support under the Prosperity Partnership Grant "Cornerstone: Mechanical Engineering Science to Enable Aero Propulsion Futures", Grant Ref: EP/R004951/1.

\section{References}

[1] Yamauchi, K., Fukui, T., and Morinishi, K., "Numerical Simulation of Influences of the Body's Presence on Flow Around the Wings in Insect Flapping Flight," ASME-JSME-KSME 2019 8th Joint Fluids Engineering Conference, San Francisco, 2019, pp. $1-8$.

Accepted manuscript: AIAA SciTech 2021 Forum, https:///doi.org/10.2514/6.2021-1663

Copyright $(0) 2021$ by the American Institute of Aeronautics and Astronautics, Inc. All rights reserved. 
[2] King, M. F., Khan, A., Delbosc, N., Gough, H. L., Halios, C., Barlow, J. F., and Noakes, C. J., "Modelling urban airflow and natural ventilation using a GPU-based lattice-Boltzmann method," Building and Environment, Vol. 125, 2017, pp. $273-284$. https://doi.org/10.1016/j.buildenv.2017.08.048, URL https://doi.org/10.1016/j.buildenv.2017.08.048

[3] Krüger, T., Kusumaatmaja, H., Kuzmin, A., Shardt, O., Silva, G., and Viggen, E. M., The Lattice Boltzmann Method - Principles and Practice, $1^{\text {st }}$ ed., Springer International Publishing, 2017. https://doi.org/10.1007/978-3-319-44649-3

[4] Dellar, P. J., "Lattice Boltzmann algorithms without cubic defects in Galilean invariance on standard lattices," Journal of Computational Physics, Vol. 259, 2014, pp. 270-283. https://doi.org/10.1016/j.jcp.2013.11.021, URL http://dx.doi.org/10. 1016/j.jcp.2013.11.021

[5] Alexander, F. J., and Chen, S., "Lattice Boltzmann thermohydrodynamics," Physical Review E, Vol. 47, No. 4, 1993.

[6] Kataoka, T., and Tsutahara, M., "Lattice Boltzmann model for the compressible Navier-Stokes equations with flexible specific-heat ratio,” Physical Review E, Vol. 69, No. 3, 2004. https://doi.org/10.1103/PhysRevE.69.035701

[7] Li, K., and Zhong, C., “A lattice Boltzmann model for simulation of compressible flows," International Journal for Numerical Methods in Fluids, Vol. 77, No. 6, 2015, pp. 334-357. https://doi.org/10.1002/fld

[8] He, X., Chen, S., and Doolen, G. D., "A Novel Thermal Model for the Lattice Boltzmann Method in Incompressible Limit," Journal of Computational Physics, Vol. 146, No. 1, 1998, pp. 282-300.

[9] Dellar, P. J., "Two routes from the Boltzmann equation to compressible flow of polyatomic gases,” Progress in Computational Fluid Dynamics, Vol. 8, No. 1-4, 2008, pp. 84-96. https://doi.org/10.1504/PCFD.2008.018081

[10] Guo, Z., Zheng, C., Shi, B., and Zhao, T. S., “Thermal lattice Boltzmann equation for low Mach number flows: Decoupling model," Physical Review E, Vol. 75, No. 3, 2007. https://doi.org/10.1103/PhysRevE.75.036704

[11] Karlin, I. V., Sichau, D., and Chikatamarla, S. S., "Consistent two-population lattice Boltzmann model for thermal flows," Physical Review E, Vol. 88, No. 6, 2013, pp. 1-13. https://doi.org/10.1103/PhysRevE.88.063310

[12] Li, Q., He, Y. L., Wang, Y., and Tao, W. Q., "Coupled double-distribution-function lattice Boltzmann method for the compressible Navier-Stokes equations,” Physical Review E, Vol. 76, No. 5, 2007. https://doi.org/10.1103/PhysRevE.76.056705.

[13] Gan, Y., Xu, A., Zhang, G., and Yang, Y., "Lattice BGK kinetic model for high-speed compressible flows: Hydrodynamic and nonequilibrium behaviors," European Physics Letters, Vol. 103, No. 2, 2013. https://doi.org/10.1209/0295-5075/103/24003

[14] Chen, F., Xu, A., Zhang, G., Gan, Y., Cheng, T., and Li, Y., "Highly Efficient Lattice Boltzmann Model for Compressible Fluids : Two-Dimensional Case," Communications in Theoretical Physics, Vol. 52, No. 4, 2009, pp. 681-693.

[15] Frapolli, N., Chikatamarla, S. S., and Karlin, I. V., "Multispeed entropic lattice Boltzmann model for thermal flows," Physical Review E, Vol. 90, No. 04, 2014, pp. 1-13. https://doi.org/10.1103/PhysRevE.90.043306

[16] Sterling, J. D., and Chen, S., "Stability Analysis of Lattice Boltzmann Methods," Journal of Computational Physics, Vol. 123, No. 1, 1996, pp. 196-206.

[17] Lallemand, P., and Luo, L. S., "Theory of the lattice Boltzmann method: Dispersion, dissipation, isotropy, Galilean invariance, and stability," Physical Review E - Statistical Physics, Plasmas, Fluids, and Related Interdisciplinary Topics, Vol. 61, No. 6, 2000, pp. 6546-6562. https://doi.org/10.1103/PhysRevE.61.6546

[18] Siebert, D. N., Hegele, L. A., and Philippi, P. C., "On lattice Boltzmann equation linear stability analysis : Thermal and athermal models," Physical Review E, Vol. 77, No. 2, 2008. https://doi.org/10.1103/PhysRevE.77.026707.

[19] Sod, G. A., “A Survey of Several Finite DIfference Methods for Systems of Nonlinear Hyperbolic Conservation Laws," Journal of Computational Physics, Vol. 27, 1978, pp. 1-31.

[20] Chapman, S., and Cowling, T., The mathematical theory of non-uniform gasses, Cambridge Univeristy Press, Cambridge, 1953.

[21] Shan, X., Yuan, X.-F., and Chen, H., "Kinetic theory representation of hydrodynamics: a way beyond the Navier-Stokes equation," Journal of Fluid Mechanics, Vol. 550, 2006, pp. 413-441. https://doi.org/10.1017/S0022112005008153

[22] Xu, A. G., Zhang, G. C., Gan, Y. B., Chen, F., and Yu, X. J., "Lattice Boltzmann modeling and simulation of compressible flows," Frontiers of Physics, Vol. 7, No. 5, 2012, pp. 582-600. https://doi.org/10.1007/s11467-012-0269-5

Accepted manuscript: AIAA SciTech 2021 Forum, https://doi.org/10.2514/6.2021-1663

Copyright (c) 2021 by the American Institute of Aeronautics and Astronautics, Inc. All rights reserved. 
[23] Warming, R. F., and Beam, R. M., "Upwind Second-Order Difference Schemes and Applications in Aerodynamic Flows," AIAA Journal, Vol. 14, No. 9, 1976, pp. 1241-1249. https://doi.org/10.2514/3.61457.

[24] Anderson, J., Fundamentals of Aerodynamics, $5^{\text {th }}$ ed., McGraw-Hill Education, 2011.

[25] Guo, Z. L., Zheng, C. G., and Shi, B., "Non-Equilibrium Extrapolation Method for Velocity and Pressure Boundary Conditions in the Lattice Boltzmann Method," Chinese Physics, Vol. 11, 2002, pp. 366-374.

Accepted manuscript: AIAA SciTech 2021 Forum, https://doi.org/10.2514/6.2021-1663

Copyright (C) 2021 by the American Institute of Aeronautics and Astronautics, Inc. All rights reserved. 